\title{
Effectiveness of empiric aztreonam compared to other beta-lactams for treatment of Pseudomonas aeruginosa infections
}

This article was published in the following Dove Press journal: Infection and Drug Resistance

\author{
Michael Hogan' \\ Mary Barna Bridgeman' \\ Gee Hee Min' \\ Deepali Dixit ${ }^{\prime}$ \\ Patrick J Bridgeman' \\ Navaneeth Narayanan ${ }^{1,2}$ \\ 'Department of Pharmacy Practice \\ and Administration, Ernest Mario \\ School of Pharmacy, Rutgers \\ University, Piscataway, NJ, USA; \\ ${ }^{2}$ Division of Infectious Diseases, \\ Department of Medicine, Rutgers \\ Robert Wood Johnson Medical School, \\ New Brunswick, NJ, USA
}

Purpose: To evaluate the use of aztreonam as an active empiric therapy against subsequent culture of Pseudomonas aeruginosa (P. aeruginosa).

Methods: This was a retrospective cohort study conducted among patients who received either aztreonam or an antipseudomonal beta-lactam (BL) as an empiric therapy with subsequent culture with $P$. aeruginosa. All patients with at least one positive culture for $P$. aeruginosa between January 2014 and August 2016 were included in this analysis. The primary composite outcome was empiric therapy failure, defined as inappropriate empiric therapy, alteration of empiric antibiotic following culture results, or 30-day in-hospital mortality. Secondary outcomes included appropriate empiric therapy, alteration of empiric therapy, 30-day-in-hospital mortality, and post-culture hospital length of stay.

Results: The primary outcome of empiric therapy failure was significantly higher in the aztreonam group than in the BL group ( $77.8 \%$ vs $41.9 \%$; $P=0.004)$. The aztreonam group had a lower rate of appropriate empiric therapy compared with the BL group (44.4\% vs $66.1 \%$; $P=0.074)$ and higher alteration of empiric therapy once susceptibilities were known than when compared with the BL group $(61.1 \% \mathrm{vs} 28.2 \% ; P=0.005)$. Although numerically higher, 30 -day-in-hospital mortality and median hospital length of stay were not significantly different between the two groups.

Conclusion: Empiric therapy failure occurred more often when initially using aztreonam vs a BL in a patient who subsequently had a P. aeruginosa infection. Only a third of patients within the aztreonam group had a documented BL allergy, demonstrating an inclination for clinicians to utilize this drug as an empiric therapy when there were more appropriate therapies available. Keywords: Pseudomonas aeruginosa, aztreonam, empiric therapy, anti-bacterial agents, beta-lactams

\section{Introduction}

Pseudomonas aeruginosa (P. aeruginosa) is a multi-drug resistant (MDR), aerobic, gram-negative bacillus bacteria. Infections caused by $P$. aeruginosa attribute to $10 \%-15 \%$ of hospital-acquired infections around the world, ${ }^{1}$ and $\sim 7 \%$ of infections in the United States. ${ }^{2}$ Although adequate treatment regimens are available to treat $P$. aeruginosa, these infections contribute to high morbidity and mortality rates. ${ }^{3}$ Mortality due to $P$. aeruginosa infections may be attributed to the high virulence of $P$. aeruginosa, increased resistance to many antimicrobials favoring inadequacy of empiric therapy, and its ability to develop resistance during therapy; patients with prolonged hospitalizations are highly susceptible to nosocomial infections due to certain comorbidities, such as immunosuppression. ${ }^{4-6}$ P. aeruginosa develops resistance through intrinsic structural
Correspondence: Mary Barna Bridgeman Department of Pharmacy Practice and Administration, Ernest Mario School of Pharmacy, Rutgers University, 160 Frelinghuysen Road, Piscataway, NJ 08854, USA

Tel +l 8484456815

Email Mary.bridgeman@pharmacy. rutgers.edu 
means (low permeable outer membrane, porin changes, and upregulation of efflux pumps) as well as the naturally occurring beta-lactamases, ${ }^{7}$ resulting in a reduction of the number of effective empiric antibiotics. The cost of an MDR strain of $P$. aeruginosa vs a nonresistant strain has shown to drastically increase admission costs. Pharmacy costs were seen to increase three-fold in these circumstances, demonstrating correct choice of antibiotics is crucial not only to patient outcomes, but also to hospital cost savings. ${ }^{8}$

Agents of choice for empiric therapy are antimicrobials that cover a broad spectrum of bacteria. Empiric therapy for patients at risk of gram-negative infection varies depending on the source of the infection and host-specific characteristics (eg, allergies and renal function). Carbapenems and later generation beta-lactams (BLs) have broad coverage and both cover P. aeruginosa as well as gram-positive bacteria. In the United States, the Food and Drug Administration (FDA)approved drugs within these classes that encompass susceptibility specifically to $P$. aeruginosa include ureidopenicillins (piperacillin), monobactams (aztreonam), later-generation cephalosporins (ceftazidime, ceftolozane, cefepime), and carbapenems (meropenem, doripenem, and imipenem). ${ }^{9}, 10$ Certain non-BLs (NBLs), including fluoroquinolones (FQ; ciprofloxacin or levofloxacin) and aminoglycosides, also cover $P$. aeruginosa and may also be used in combination with $\mathrm{BL}$ agents as empiric therapy in select clinical situations. ${ }^{10}$ The aforementioned agents are consistent with recommendations from the 2016 Infectious Disease Society of America (IDSA) guidelines on the management of adults with hospital-acquired or ventilator-associated pneumonia with a caveat that agent selection be based on routinely updated local antibiograms. Notably, monotherapy with antipseudomonal activity suffices as long as local resistance does not exceed $10 \%$ for the selected agent. ${ }^{11}$

Treatment with a BL for empiric therapy can sometimes be challenging due to the high percentage of patients with documented or self-reported penicillin or cephalosporin allergies. Overall, reported incidence of a BL allergy for hospitalized patients is $\sim 17.3 \%{ }^{12}$ Not all patient-reported penicillin allergies are restrictive to BL therapy; in fact, up to $90 \%$ of patients who have reported penicillin allergies have been shown to tolerate other BLs. ${ }^{13-16}$ Although skin testing exists to confirm patient-reported allergies, it is not generally recommended to perform in patients that have active infections due to evidence that delaying empiric therapy leads to increased mortality in those patients. ${ }^{17-19}$

An acceptable alternative for patients with suspected P. aeruginosa infection with a severe BL allergy is to utilize the only FDA-approved monobactam, aztreonam..$^{20,21}$ Aztreonam is a bactericidal agent that acts by inhibition of bacterial cell wall synthesis. ${ }^{22}$ It has high affinity for the penicillin-binding protein 3 of gram-negative bacteria resulting in broad spectrum activity against gram negatives, but no activity against gram-positive and anaerobic bacteria, very similar to aminoglycosides, but with improved safety. ${ }^{23}$ However, it is not the most attractive option for an initial monotherapy due to its narrow coverage. The greatest utility of aztreonam is for narrowed treatment of infections caused by gram-negative aerobic bacteria susceptible to aztreonam in patients with severe allergy to penicillin or other BLs. ${ }^{24}$

The purpose of this study was to assess clinical outcomes of patients who receive aztreonam as an empiric therapy for the intended treatment of $P$. aeruginosa infections compared with traditional antipseudomonal BL empiric therapy.

\section{Methods}

\section{Setting and study population}

We conducted a retrospective cohort study of all inpatients admitted from January 2014 to August 2016 at a tertiary care academic medical center. Patients $>18$ years old with at least one positive culture for $P$. aeruginosa who were treated as such with either aztreonam or other antipseudomonal BL therapy (ceftazidime, ceftolozane/tazobactam, cefepime, piperacillin/tazobactam, meropenem, imipenem, and doripenem) as empiric monotherapy to treat $P$. aeruginosa for $\geq 48$ hours were included. Patients were identified through the hospital microbiology laboratory database, and further clinical data were abstracted from the hospital electronic medical record (EMR). Only the first infection/ treatment episode per patient was recorded and analyzed by assessing the first sample for patients with more than one positive $P$. aeruginosa culture. Patients were excluded if they were being treated with a FQ or NBL therapy, were on dual therapy, and were only prescribed targeted therapy after culture susceptibility results known, were pregnant, or were treated as outpatients. The Rutgers University Institutional Review Board approved this study and determined patient consent for medical record review was not required based on the retrospective nature of this study; deidentified patient data were collected and analyzed.

\section{Predictor and covariates}

The primary predictor of interest was the use of empiric aztreonam therapy with a control comparison of other BLs with antipseudomonal activity. Data on multiple demographic and clinical covariates were collected including age, sex, 
weight, severity of illness (measured by Sequential Organ Failure Assessment Score [SOFA] score), comorbidities (measured by Charlson Comorbidity index), presence of documented BL allergy, and source of culture positive for P. aeruginosa. Empiric therapy was confirmed by reviewing the EMR and assessing when the positive $P$. aeruginosa culture was collected and time until first dose administration of either aztreonam or a BL.

\section{Outcomes measures}

The primary composite outcome was empiric therapy failure, defined as inappropriate empiric therapy, alteration of empiric antibiotic following culture results, or 30-day in-hospital mortality. Secondary outcomes included appropriate empiric therapy, defined as receipt of an antibiotic with in-vitro coverage of the cultured pathogen (P. aeruginosa) prior to culture/ sensitivity results, alteration of empiric therapy, defined as change of antibiotic therapy following culture results, 30-day in-hospital mortality, and post-culture hospital length of stay (LOS).

\section{Statistical analysis}

Descriptive statistics were utilized to describe all variables. Continuous data were reported as the median and interquartile range (IQR). All categorical variables were reported as percentages. The Mann-Whitney $U$-test was used to test for comparison of nonparametric continuous variables. The chisquare test was used for comparison of categorical variables. A two-sided significance level of $P<0.05$ was set a priori.

The primary relationship of interest is the association of empiric aztreonam therapy and empiric therapy failure. We performed a bivariate logistic regression analysis on measured covariates to assess for possible confounders. Any variable in the bivariate analysis with $P<0.2$ and deemed clinically relevant was entered into a multivariate logistic regression model to adjust for potential confounding effects. An adjusted OR (aOR) and 95\% CI were calculated to determine the magnitude of association of empiric aztreonam and empiric therapy failure while controlling for confounders. Data analysis was performed using Stata, version 15.0 (StataCorp).

\section{Results}

\section{Patient population}

During the study period, 636 positive cultures of $P$. aeruginosa were screened for eligibility. A total of 142 patients met the inclusion criteria with $18(12.7 \%)$ patients in the aztreonam group and $124(87.3 \%)$ patients in the BL group. Majority of patients excluded were those who received only targeted antibiotic therapy based on culture results, not empiric therapy, followed by patients who were not prescribed any antimicrobial treatment for a positive culture. Most patients in the control group were ordered to receive empiric piperacillin-tazobactam therapy (98/124).

Baseline characteristics between groups were similar (Table 1). The median age of patients was 72.5 years (IQR, 64.9-83.5 years) in the aztreonam group and 67.4 years (IQR, 55.3-76.4 years) in the BL group $(P=0.026)$. The majority of patients in each group were male $(61.1 \%$ vs $58.9 \% ; P=0.857)$. Source of infections were similar between groups, with respiratory and urine sources making up more than half of patients.

Patients were more severely ill in the aztreonam group than in the BL group according to SOFA scores, with a median score of 9.5 (IQR, 4-14) in the aztreonam group and

Table I Baseline characteristics

\begin{tabular}{|c|c|c|c|}
\hline Characteristic & $\begin{array}{l}\text { Other Anti-PsA BL } \\
(n=124)\end{array}$ & $\begin{array}{l}\text { Aztreonam } \\
(n=18)\end{array}$ & $P$-value \\
\hline Age (years), median (IQR) & $67.4(55.3-76.4)$ & $72.5(64.9-83.5)$ & 0.026 \\
\hline Male, n (\%) & $73(58.9)$ & $I I(6 I . I)$ & 0.857 \\
\hline Weight (kg), median (IQR) & $73.3(62.8-89.3)$ & $76.7(70.3-94.1)$ & 0.265 \\
\hline SOFA score, median (IQR) & $6.5(3-11)$ & $9.5(4-14)$ & 0.216 \\
\hline Charlson Comorbidity Index, median (IQR) & $7(4-10)$ & $8(7-10)$ & 0.117 \\
\hline Documented beta-lactam allergy, n (\%) & $4(3.2)$ & $7(38.9)$ & $<0.001$ \\
\hline \multicolumn{3}{|l|}{ Source of culture, n (\%) } & 0.151 \\
\hline Blood & $13(10.5)$ & I (5.6) & \\
\hline Respiratory & $52(4 I .9)$ & $7(38.9)$ & \\
\hline Urine & $27(21.8)$ & $4(22.2)$ & \\
\hline Tissue/wound & $21(16.9)$ & $\mathrm{I}(5.6)$ & \\
\hline Other & II (8.9) & $5(27.8)$ & \\
\hline
\end{tabular}

Notes: Other Anti-PsA BL includes piperacillin-tazobactam ( $n=98)$, meropenem $(n=15)$, ceftazidime $(n=8)$, and cefepime $(n=3)$.

Abbreviations: Anti-PsA BL, anti-pseudomonal beta-lactam; IQR, interquartile range; SOFA, sequential organ failure assessment. 
6.5 (IQR, 3-11) in the $\mathrm{BL}$ group $(P=0.216$ ). A significantly higher number of patients had documented BL allergy in the aztreonam group than in the BL group (38.9\% vs 3.2\%; $P<0.001)$.

\section{Outcomes}

The primary outcome of empiric therapy failure was significantly higher in the aztreonam group than in the BL group (77.8\% vs $41.9 \%$; $P=0.004$; Table 2 ). The aztreonam group had a lower rate of appropriate empiric therapy (44.4\%) compared with the BL group $(66.1 \%, P=0.074)$ and significantly higher alteration of empiric therapy (61.1\%) compared with the $\mathrm{BL}$ group $(28.2 \%, P=0.005)$. Although numerically higher, 30-day in-hospital mortality and median post-culture draw hospital LOS were not statistically significantly different between the two groups. In multivariate logistic regression analysis, the odds of empiric therapy failure were significantly higher among patients who received aztreonam as an empiric therapy when compared with those who received other BL therapy (aOR 6.15; 95\% CI 1.56, 24.33) after adjusting for SOFA score and source of positive $P$. aeruginosa culture (Table 3).

\section{Discussion}

This study was conducted with the intent of assessing the effectiveness of empiric aztreonam therapy for patients with subsequent cultures identifying $P$. aeruginos $a$ when compared with standard antipseudomonal BL therapy. Of note, investigators observed patients receiving empiric aztreonam to be generally older with higher severity of illness, according to SOFA scores; we hypothesize this because aztreonam is generally a more conservative selection in patients with even a remote history of penicillin allergy. We observed that those patients receiving empiric aztreonam had a significantly higher rate of empiric therapy failure compared with use of other therapies. After adjusting for confounding factors, aztreonam remained significantly associated with empiric therapy failure, with higher odds when compared with standard antipseudomonal BL empiric therapy. The primary composite outcome findings are driven by the significantly higher rate of alteration of empiric therapy by the treating clinician following culture and sensitivity results and numerically higher inappropriate therapy and in-hospital mortality. In addition to the main findings, it is important to note that $<40 \%$ of patients treated empirically with aztreonam had documented $\mathrm{BL}$ allergies, suggesting that another more effective antipseudomonal BL could have been used, although improper allergy documentation limits definitive conclusions regarding the use of first-line therapy.

National guidelines from the IDSA cautiously recommend empiric aztreonam as an initial therapy for a patient with suspected P. aeruginosa infection. ${ }^{11}$ We have observed clinicians at our institution prescribing aztreonam monotherapy as an empiric therapy to cover for gram-negative pathogens; however, based on our study results, we observed a high likelihood of empiric therapy failure when cultures grow $P$. aeruginosa.

There are limited data available on the use of aztreonam as an empiric therapy to cover $P$. aeruginosa. One study, conducted in 1993, compared empiric aztreonam to

Table 2 Clinical outcome results

\begin{tabular}{|c|c|c|c|}
\hline Study outcome & $\begin{array}{l}\text { Other Anti-PsA BL } \\
(n=124)\end{array}$ & $\begin{array}{l}\text { Aztreonam } \\
(n=18)\end{array}$ & $P$-value \\
\hline Empiric therapy failure, $n(\%)$ & $52(4 I .9)$ & $14(77.8)$ & 0.004 \\
\hline Alteration of empiric therapy, $\mathrm{n}(\%)$ & $35(28.2)$ & $1 \mathrm{I}(6 \mathrm{I} . \mathrm{I})$ & 0.005 \\
\hline Appropriate empiric therapy, $\mathrm{n}(\%)$ & $82(66.1)$ & $8(44.4)$ & 0.074 \\
\hline Thirty-day in-hospital mortality, n (\%) & $8(6.5)$ & $3(16.7)$ & 0.13 \\
\hline Post-culture draw hospital LOS, median (IQR) & $13(7-21.5)$ & $13.5(10-27)$ & 0.229 \\
\hline
\end{tabular}

Notes: Other Anti-PsA BL includes piperacillin-tazobactam $(n=98)$, meropenem $(n=15)$, ceftazidime $(n=8)$, and cefepime $(n=3)$.

Abbreviations: Anti-PsA BL, anti-pseudomonal beta-lactam; IQR, interquartile range; LOS, length of stay.

Table 3 Logistic regression analysis assessing association of empiric aztreonam therapy and empiric therapy failure

\begin{tabular}{|l|l|l|}
\hline Characteristic & $\begin{array}{l}\text { Unadjusted analysis } \\
\text { Crude OR (95\% CI) }\end{array}$ & $\begin{array}{l}\text { Multivariable analysis } \\
\text { Adjusted }^{\text {OR }} \text { (95\% CI) }\end{array}$ \\
\hline Empiric aztreonam therapy & $4.85(\mathrm{I} .5 \mathrm{I}, \mathrm{I5.57)}$ & $6.15(\mathrm{I.56,24.33)}$ \\
\hline Other Anti-PsA BL therapy & Reference & Reference \\
\hline
\end{tabular}

Note: ${ }^{a}$ Adjusted for SOFA score and source of positive Pseudomonas aeruginosa culture.

Abbreviations: Anti-PsA BL, anti-pseudomonal beta-lactam; SOFA, sequential organ failure assessment. 
aminoglycoside therapy in the treatment of serious lower respiratory infections; most bacteria isolated in this study were Enterobacteriaceae sp. (44\%), with $19 \%$ of patients found to have $P$. aeruginosa infections. Overall, positive results came from this study, with $72 \%$ eradication from the aztreonam group vs $57 \%$ from the control group $(P=0.357)$ with shorter hospital stays in the aztreonam group. This study was conducted closer to aztreonam's FDA approval date (1986), with less bacterial exposure than it has now to P. aeruginosa, so resistance patterns cannot be extrapolated. This study also shows the value that, although similar in efficacy to aminoglycosides, aztreonam was better tolerated with favorable safety data. ${ }^{23}$

There are conflicting mortality results when evaluating inappropriate initial empiric antibiotic selection. Patients who are undoubtedly sicker, including those on ventilators, ${ }^{25}$ and those with sepsis, ${ }^{26-29}$ showed increased mortality when inappropriate empiric therapy was utilized. Other studies found no significance in mortality risk when looking at inappropriate empiric therapy for gram-positive and gram-negative organisms. ${ }^{29,30}$ One study had similar results to our study when looking at inappropriate empiric therapy with regard to P. aeruginosa. Investigators found, in general, that inappropriate empiric therapy did not lead to higher mortality; however, when evaluated according to specific sites of infection, there was an association with respiratory and intraabdominal infections cultured with $P$. aeruginos $a$ and mortality. ${ }^{31}$

In our study, we see only one-third of patients with reported BL allergies were treated with aztreonam. A study with a similar study design as ours retrospectively evaluated patients with severe BL allergies receiving either a BL or an NBL (including aztreonam). They found that, despite a slight risk of hypersensitivity reactions occurring with the use of a $\mathrm{BL}$ in documented BL-allergic patients, there was a significant reduction in clinical failure at 72-96 hours. ${ }^{32}$ Approximately $80 \%$ of patients with a BL allergy still received a BL and hypersensitivity rates were low. This study gives clinicians some evidence that using BL in patients with a BL allergy may be appropriate to treat a patient most effectively. ${ }^{32}$

One of the major limitations of this study is the small sample size of aztreonam-treated patients over the 2 years, specifically for $P$. aeruginosa. Our institution implemented an antimicrobial stewardship restriction on aztreonam starting in 2015 , in the middle of the retrospective study period. This meant that there needed to be permission in order to prescribe empiric aztreonam after 2015 or documentation of a severe BL allergy. This could drastically reduce the number of cases where aztreonam could still be used as an empiric therapy; additionally, authors suggest the results of this analysis be interpreted with caution due to the limited sample size. Another limitation was the lack of descriptive data toward BL allergies. The EMR system reported only whether they were allergic to penicillin with not much description, so we may have underrepresented patients with BL allergies. Finally, although necessary for independence of observations, we looked only at the first hospital stay of a patient who was readmitted multiple times within the 2 years, which may have caused some missed cases of patients treated empirically with aztreonam.

Despite these limitations, the findings of this retrospective analysis suggest that, with limitations, when using aztreonam as an empiric therapy in a patient subsequently found to have a $P$. aeruginosa infection, empiric therapy failure occurred more often than for patients treated with using a standard antipseudomonal BL. Approximately, one-third of patients within the aztreonam group had a documented BL allergy, demonstrating an inclination of clinicians to use this drug as an empiric therapy despite the availability of more appropriate therapies.

\section{Conclusion}

Although definitive conclusions cannot be made based on the results of this analysis, this study reinforces antimicrobial stewardship principles, including proper assessment of patients with BL allergies to promote use of first-line agents for treatment of infectious diseases. ${ }^{33}$ The use of aztreonam should be reserved for patients with severe BL allergies, since more appropriate alternative antimicrobial options exist that can be safely administered following evaluation and clinical confirmation of the reported allergy. ${ }^{34}$ Additional studies with larger sample sizes and direct comparisons to specific BL agents could support these findings. These data can be used by clinicians to aid in the choice of empiric therapy for a patient with a suspected gram-negative bacterial infection, more specifically P. aeruginosa, to improve clinical outcomes.

\section{Key points}

1. The results of this study suggest that empiric aztreonam use led to increased empiric therapy failure in patients with $P$. aeruginosa infection.

2. More than half of clinicians $(61.1 \%)$ changed therapy for those patients receiving aztreonam as an empiric therapy once cultures and sensitivities were known.

3. Little more than half $(55.6 \%)$ of patients who were given aztreonam empirically were considered inappropriate or were resistant to aztreonam once susceptibilities were known. 


\section{Disclosure}

The authors report no conflicts of interest in this work.

\section{References}

1. Blanc DS, Petignat C, Janin B, Bille J, Francioli P. Frequency and molecular diversity of Pseudomonas aeruginosa upon admission and during hospitalization: a prospective epidemiologic study. Clin Microbiol Infect. 1998;4(5):242-247.

2. Magill SS, Edwards JR, Bamberg W, et al. Multistate point-prevalence survey of health care-associated infections. $N$ Engl J Med. 2014;370(13):1198-1208.

3. Bassetti M, Righi E, Viscoli C. Pseudomonas aeruginosa serious infections: mono or combination antimicrobial therapy? Curr Med Chem. 2008;15(5):517-522.

4. Harris A, Torres-Viera C, Venkataraman L, Degirolami P, Samore M, Carmeli Y. Epidemiology and clinical outcomes of patients with multiresistant Pseudomonas aeruginosa. Clin Infect Dis. 1999;28(5):1128-1133.

5. Kang CI, Kim SH, Kim HB, et al. Pseudomonas aeruginosa bacteremia: risk factors for mortality and influence of delayed receipt of effective antimicrobial therapy on clinical outcome. Clin Infect Dis. 2003;37(6):745-751.

6. Osih RB, Mcgregor JC, Rich SE, et al. Impact of empiric antibiotic therapy on outcomes in patients with Pseudomonas aeruginosa bacteremia. Antimicrob Agents Chemother. 2007;51(3):839-844.

7. Nordmann P, Guibert M. Extended-spectrum beta-lactamases in Pseudomonas aeruginosa. J Antimicrob Chemother. 1998;42(2): $128-131$.

8. Morales E, Cots F, Sala M, et al. Hospital costs of nosocomial multidrug resistant Pseudomonas aeruginosa acquisition. BMC Health Serv Res. 2012;12:122.

9. Pechère JC, Köhler T. Patterns and modes of beta-lactam resistance in Pseudomonas aeruginosa. Clin Microbiol Infect. 1999;5(Suppl 1):S15-S18.

10. Gilbert DN, Chambers HF, Eliopoulos GM, et al. The Sanford Guide to Antimicrobial Therapy 2018. 48th edition. Sperryville, VA: Antimicrobial Therapy, Inc,; 2018.

11. Kalil AC, Metersky ML, Klompas M, et al. Executive summary: management of adults with hospital-acquired and ventilator-associated pneumonia: 2016 Clinical Practice Guidelines by the Infectious Diseases Society of America and the American Thoracic Society. Clin Infect Dis. 2016;63(5):575-582.

12. Lee CE, Zembower TR, Fotis MA, et al. The incidence of antimicrobial allergies in hospitalized patients: implications regarding prescribing patterns and emerging bacterial resistance. Arch Intern Med. 2000;160(18):2819-2822.

13. Macy E, Burchette RJ. Oral antibiotic adverse reactions after penicillin skin testing: multi-year follow-up. Allergy. 2002;57(12):1151-1158.

14. Macy E, Ngor EW. Safely diagnosing clinically significant penicillin allergy using only penicilloyl-poly-lysine, penicillin, and oral amoxicillin. J Allergy Clin Immunol Pract. 2013;1(3):258-263.

15. Sogn DD, Evans R, Shepherd GM, et al. Results of the national institute of allergy and infectious diseases collaborative clinical trial to test the predictive value of skin testing with major and minor penicillin derivatives in hospitalized adults. Arch Intern Med. 1992;152(5): $1025-1032$.

16. Gadde J, Spence M, Wheeler B, Adkinson NF. Clinical experience with penicillin skin testing in a large inner-city STD clinic. JAMA. 1993;270(20):2456-2463.
17. Gaieski DF, Mikkelsen ME, Band RA, et al. Impact of time to antibiotics on survival in patients with severe sepsis or septic shock in whom early goal-directed therapy was initiated in the emergency department. Crit Care Med. 2010;38(4):1045-1053.

18. Kumar A, Roberts D, Wood KE, et al. Duration of hypotension before initiation of effective antimicrobial therapy is the critical determinant of survival in human septic shock. Crit Care Med. 2006;34(6):1589-1596.

19. Morrell M, Fraser VJ, Kollef MH. Delaying the empiric treatment of candida bloodstream infection until positive blood culture results are obtained: a potential risk factor for hospital mortality. Antimicrob Agents Chemother. 2005;49(9):3640-3645.

20. Buonomo A, Nucera E, de Pasquale T, et al. Tolerability of aztreonam in patients with cell-mediated allergy to $\beta$-lactams. Int Arch Allergy Immunol. 2011;155(2):155-159.

21. Patriarca G, Schiavino D, Lombardo C, et al. Tolerability of aztreonam in patients with IgE-mediated hypersensitivity to beta-lactams. Int $J$ Immunopathol Pharmacol. 2008;21(2):375-379.

22. Azactam ${ }^{\circledR}$ (aztreonam injection) package insert. Princeton, NJ: BristolMyers Squibb Company; 2013.

23. Bjornson HS, Ramirez-Ronda C, Saavedra S, Rivera-Vázquez CR, Liu $\mathrm{C}$, Hinthorn DR. Comparison of empiric aztreonam and aminoglycoside regimens in the treatment of serious gram-negative lower respiratory infections. Clin Ther. 1993;15(1):65-78.

24. Doi Y, Chambers HF. Other B-lactam antibiotics. In: Bennett J.E., editor. Mandell, Douglas, and Bennett's Principles and Practice of Infectious Diseases. 8th edition. Pennsylvania: Saunders; 2015:293-297.

25. Torres MJ, Mayorga C, Leyva L, et al. Controlled administration of penicillin to patients with a positive history but negative skin and specific serum IgE tests. Clin Exp Allergy. 2002;32(2):270-276.

26. Paul M, Zemer-Wassercug N, Talker O, et al. Are all beta-lactams similarly effective in the treatment of methicillin-sensitive Staphylococcus aureus bacteraemia? Clin Microbiol Infect. 2011;17(10):1581-1586.

27. Vallés J, Rello J, Ochagavía A, Garnacho J, Alcalá MA. Communityacquired bloodstream infection in critically ill adult patients: impact of shock and inappropriate antibiotic therapy on survival. Chest. 2003;123(5):1615-1624.

28. Ibrahim EH, Sherman G, Ward S, Fraser VJ, Kollef MH. The influence of inadequate antimicrobial treatment of bloodstream infections on patient outcomes in the ICU setting. Chest. 2000;118(1):146-155.

29. Kim SH, Park WB, Lee KD, et al. Outcome of inappropriate initial antimicrobial treatment in patients with methicillin-resistant Staphylococcus aureus bacteraemia. J Antimicrob Chemother. 2004;54(2):489-497.

30. Frakking FN, Rottier WC, Dorigo-Zetsma JW, et al. Appropriateness of empirical treatment and outcome in bacteremia caused by extended-spectrum- $\beta$-lactamase-producing bacteria. Antimicrob Agents Chemother. 2013;57(7):3092-3099.

31. Joo EJ, Kang CI, Ha YE, et al. Risk factors for mortality in patients with Pseudomonas aeruginosa bacteremia: clinical impact of antimicrobial resistance on outcome. Microb Drug Resist. 2011;17(2):305-312.

32. Jeffres MN, Narayanan PP, Shuster JE, Schramm GE. Consequences of avoiding $\beta$-lactams in patients with $\beta$-lactam allergies. $J$ Allergy Clin Immunol. 2016;137(4):1148-1153.

33. Barlam TF, Cosgrove SE, Abbo LM, et al. Implementing an antibiotic stewardship program: Guidelines by the Infectious Diseases Society of America and the Society for Healthcare Epidemiology of America. Clin Infect Dis. 2016;62(10):e51-e 77.

34. Joint Task Force on Practice Parameters; American Academy of Allergy, Asthma and Immunology; American College of Allergy, Asthma and Immunology; Joint Council of Allergy, Asthma and Immunology. Drug allergy: an updated practice parameter. Ann Allergy Asthma Immunol. 2010;105:259-273. 
Infection and Drug Resistance is an international, peer-reviewed openaccess journal that focuses on the optimal treatment of infection (bacterial, fungal and viral) and the development and institution of preventive strategies to minimize the development and spread of resistance. The journal is specifically concerned with the epidemiology of antibiotic resistance and the mechanisms of resistance development and diffusion in both hospitals and the community. The manuscript management system is completely online and includes a very quick and fair peerreview system, which is all easy to use. Visit http://www.dovepress.com/ testimonials.php to read real quotes from published authors.

Submit your manuscript here: https://www.dovepress.com/infection-and-drug-resistance-journal 